EDITORIAL

\title{
Heart failure: statins for all?
}

A F Leite-Moreira, P Castro-Chaves

Heart 2006;92:1537-1538. doi: 10.1136/hrt.2006.089797

\begin{abstract}
Although there is increasing evidence of benefit in using statins to treat patients with non-ischaemic heart failure, it is not yet possible to recommend the routine use of these drugs in all heart failure patients, irrespective of the aetiology
\end{abstract}

See end of article for authors' affiliations ......................

Correspondence to: Professor Adelino LeiteMoreira, Department of Physiology, Faculty of Medicine, University of Porto, Alameda Prof. Hernâni Monteiro, 4200

319 Porto, Portugal; amoreira@med.up.pt

Published Online First 10 April 2006
$\mathrm{T}$ he endothelium is an essential structural and functional element of the cardiovascular system and constitutes the largest endocrine system in the organism. It contributes to cardiovascular homeostasis not only by regulating vascular permeability but also by adjusting the calibre of blood vessels to haemodynamic and hormonal demands and by maintaining blood fluidity. Endothelial cells perform these functions by the expression, activation, and release of powerful vasoactive substances as well as of numerous other bioactive molecules. Almost all conventional risk factors for atherosclerosis are associated with endothelial dysfunction. Cardiovascular risk factors activate a number of pro-oxidative genes in the vascular wall resulting in the generation of reactive oxygen species that ultimately promote endothelial release of transcriptional and growth factors, proinflammatory cytokines, chemoattractant substances and adhesion molecules. ${ }^{1}$ This complex cascade of events underlies the transition from normal endothelial function to endothelial dysfunction that manifests itself by abnormal vasomotor activity, development of a procoagulant endothelial surface, inflammation and, finally, plaque formation.

One of the earliest manifestations of increased vascular oxidant stress and endothelial dysfunction is a decreased production and/or local bioavailability of nitric oxide. Clinical measurements of endothelium-dependent vasodilation by a variety of different methodologies provide a marker of endothelial integrity and are an "excellent barometer" of vascular health that can be used to gauge cardiovascular risk. ${ }^{2}$ In fact, several studies indicate that endothelial dysfunction, assessed as impaired endothelium-dependent flow-mediated vasodilation (FMD), predicts an increased rate of adverse cardiovascular events, including acute coronary syndromes, ischaemic stroke, critical limb ischaemia, coronary and carotid revascularisation procedures, and cardiovascular deaths. ${ }^{34}$

The 3-hydroxy-3-methylglutaryl-CoA (HMGCoA) reductase inhibitors, or statins, improve endothelial function and decrease plasma concentrations of tumour necrosis factor- $\alpha$ (TNF- $\alpha$ ) in patients with coronary artery disease and hyperlipidaemia. In these patients, statins have also been shown to reduce morbidity and mortality. ${ }^{5}$ Consequently, the greatest potential benefit of statins in chronic heart failure is probably in those patients with coronary artery disease. However, definitive evidence for statin treatment in patients with normocholesterolaemic non-ischaemic chronic heart failure remains to be established. In addition, there is lack of safety data for the use of statins in patients with heart failure, where there are potential concerns about coenzyme Q10 depletion and excessive low density lipoprotein (LDL) reduction that may allow unbound endotoxin to activate immune cells to produce proinflammatory cytokines. ${ }^{6}$

\section{STATINS AND ENDOTHELIAL FUNCTION}

In the study by Strey et $\mathrm{al}^{7}$ in this issue of Heart, the authors investigated and gathered evidence that short-term statin treatment improves endothelial function and neurohumoral imbalance in normocholesterolaemic patients with non-ischaemic heart failure. The study had a randomised, double-blind, placebo-controlled, crossover design and enrolled 24 patients with non-ischaemic symptomatic heart failure with reduced left ventricular ejection fraction that randomly received atorvastatin $40 \mathrm{mg}$ or placebo once daily for six weeks. Compared with placebo, statin treatment was associated with significantly greater endothelium-dependent dilation in conduit and resistance vessels, but not endothelium-independent dilation. With regard to endothelial markers and cardio-endocrine hormones the results only reached statistical significance for endothelin-1 and atrial natriuretic peptide, whose values were significantly reduced with statin treatment. These changes, however, did not have any significant effects on clinical and haemodynamic measurements of cardiac function. Although the present study may have some limitations related to the number of patients enrolled, the short follow-up period and study design, it adds to our knowledge about the beneficial effects of statins in heart failure in terms of endothelial function, neurohumoral balance and cardiac symptoms and function. Some of these beneficial effects were detected after a treatment period of just six weeks.

\section{BENEFITS OF STATINS IN HEART FAILURE}

The benefits of statins in heart failure are supported by experimental and clinical studies. Statins were originally designed to lower plasma cholesterol. However, the idea that statins mediate effects beyond cholesterol lowering,

Abbreviations: FMD, flow-mediated vasodilation; LDL, low density lipoprotein; HMG-CoA, 3-hydroxy-3methylglutaryl-CoA; TNF- $\alpha$, tumour necrosis factor- $\alpha$ 
namely through the so-called pleiotropic effects, is gaining increased strength. For example, the reduction in coronary events observed in a timeframe of just 16 weeks is too short to ascribe to the positive effects of cholesterol lowering alone. ${ }^{8}$ Statins may retard the progression of chronic heart failure through effects that may directly impact on coronary ischaemia, namely restoration of endothelial function and stabilisation of plaque, but also by additional properties such as effects on myocardial function, downregulation of AT-1 receptor, and inhibition of proinflammatory cytokines. ${ }^{9}$

In animal models of ischaemic heart failure after myocardial infarction, fluvastatin has been found to decrease left ventricular cavity dilation, myocyte hypertrophy, interstitial fibrosis and animal mortality. Although the initial phenomenon inducing heart failure was ischaemic in nature, the progression of the disease, which was significantly altered, was not dependent on further ischaemic events, supporting the non-coronary actions of these drugs in the myocardium. ${ }^{10}$

Furthermore, evidence in favour of the therapeutic benefits of statin treatment in patients with non-ischaemic heart failure, irrespective of serum cholesterol values or atherosclerotic disease, has been recently provided by several authors. In a study published by Node et al, ${ }^{11}$ including 63 patients with idiopathic dilated cardiomyopathy randomly assigned to treatment with simvastatin or placebo for 14 weeks, the authors observed an improvement in left ventricular ejection fraction, a reduction in plasma concentrations of TNF- $\alpha$, interleukin- 6 and brain natriuretic peptide and an improvement in flow-mediated brachial artery vasodilation. Multivariate analysis of changes in LDL cholesterol values showed that serum cholesterol was not a significant predictor of statin-induced improvement in endothelium-dependent vasodilation in patients with heart failure. These findings suggest that statins may improve cardiac function, in part, by modulating the inflammatory state.

\section{DIASTOLIC HEART FAILURE}

These beneficial effects may also be observed in patients with heart failure and a normal ejection fraction-that is, diastolic heart failure-as shown in a preliminary study published by Fukuta et al. ${ }^{12}$ These authors evaluated a cohort of symptomatic patients with an ejection fraction $>50 \%$ during a mean follow-up period of 21 (SD 12) months and found that, after adjustment for differences in baseline clinical variables, statin treatment was associated with lower mortality and improved survival. In a retrospective study in a cohort of elderly patients between 66-85 years with newly diagnosed heart failure, statin use within 90 days following the diagnosis was associated with a lower risk of death. ${ }^{13}$ More recently, Sola et al evaluated the effects of randomly assigned atorvastatin $20 \mathrm{mg}$ or placebo during a 12 month period in 108 patients with non-ischaemic heart failure and reduced left ventricular ejection fraction. ${ }^{14}$ These authors found that atorvastatin treatment was associated with an increase in left ventricular ejection fraction and reduced left ventricular enddiastolic diameter and end-systolic diameter. There was also an increase in erythrocyte superoxide dismutase activity and a decrease in serum concentrations of high sensitivity C-reactive protein, interleukin- 6 and TNF- $\alpha$ receptor II.

Similar results in systemic inflammation were reported in a randomised, placebo-controlled, crossover study using atorvastatin $10 \mathrm{mg}$ for 16 weeks in patients with non-ischaemic heart failure. ${ }^{15}$ However, in another study by Bleske BR et al ${ }^{16}$ these authors found a neutral effect of high dose (80 mg) atorvastatin for 12 weeks on 15 patients on markers of inflammation and endothelial activation like high sensitivity C-reactive protein, TNF- $\alpha$ soluble receptor, TNF- $\alpha$, intercellular adhesion molecule-1, P-selectin, and FMD. It has been proposed that differences in heart failure severity and statin dose used among studies might explain the distinct responses observed..$^{16}$

\section{CONCLUSION}

Although there is increasing evidence in favour of a beneficial effect of statin treatment in patients with non-ischaemic heart failure, the studies performed so far are limited by the reduced number of patients enrolled and the short period of follow-up. Thus, at the present time it is not possible to recommend the routine use of this class of drugs in all heart failure patients irrespective of the aetiology. Fortunately there are ongoing studies like CORONA (Controlled Rosuvastatin Multinational Trial in Heart Failure), GISSIHF (Gruppo Italiano per lo Studio della Sopravvivenza nell'a Insuficienza Cardiaca) or UNIVERSE (Rosuvastatin Impact on Ventricular Remodeling Lipids and Cytokines) that may help us clarify these issues in the near future.

\section{ACKNOWLEDGEMENTS}

Supported by grants from 'Fundação para a Ciência e Tecnologia' (POCI/SAU-MMO/61547/2004; partially funded by Fundo Europeu de Desenvolvimento Regional) and from 'Cardiovascular R\&D Unit' (51/94-FCT, Portugal).

\section{Authors' affiliations}

A F Leite-Moreira, P Castro-Chaves, Department of Physiology, Faculty of Medicine, University of Porto, Porto, Portugal

\section{REFERENCES}

1 Cohn JN, Quyyumi AA, Hollenberg NK, et al. Surrogate markers for cardiovascular disease: functional markers. Circulation 2004;109/25 suppl 1):IV31-46.

2 Moens AL, Goovaerts I, Claeys MJ, et al. Flow-mediated vasodilation: a diagnostic instrument, or an experimental tool? Chest 2005;127:2254-63.

3 Schachinger V, Britten MB, Zeiher AM. Prognostic impact of coronary vasodilator dysfunction on adverse long-term outcome of coronary hear disease. Circulation 2000;101:1899-906.

4 Gokce N, Keaney JF Jr, Hunter LM, et al. Predictive value of noninvasively determined endothelial dysfunction for long-term cardiovascular events in patients with peripheral vascular disease. J Am Coll Cardiol 2003;41:1769-75.

5 Long-term Intervention with Pravastatin in Ischaemic Disease (LIPID) Study Group. Prevention of cardiovascular events and death with pravastatin in patients with coronary heart disease and a broad range of initial cholesterol levels. N Engl J Med 1998;339:1349-57

6 Rauchhaus M, Coats AJ, Anker SD. The endotoxin-lipoprotein hypothesis. Lancet 2000;356:930-3.

7 Strey $\mathrm{CH}$, Young JM, Lainchbury $\mathrm{JH}$, et al. Short-term statin treatment improves endothelial function and neurohormonal imbalance in normocholesterolaemic patients with non-ischaemic heart failure. Heart 2006;92:1603-9.

8 von Haehling S, Anker SD. Statins for heart failure: at the crossroads between cholesterol reduction and pleiotropism? Heart 2005;91:1-2.

9 Krum H, McMurray JJ. Statins and chronic heart failure: do we need a largescale outcome trial? J Am Coll Cardiol 2002;39:1567-73.

10 Hayashidani S, Tsutsui H, Shiomi T, et al. Fluvastatin, a 3-hydroxy-3methylglutaryl coenzyme A reductase inhibitor, attenuates left ventricular remodeling and failure after experimental myocardial infarction. Circulation 2002; 105:868-73.

11 Node K, Fujita $M$, Kitakaze $M$, et al. Short-term statin therapy improves cardiac function and symptoms in patients with idiopathic dilated cardiomyopathy. Circulation 2003; 108:839-43.

12 Fukuta H, Sane DC, Brucks S, et al. Statin therapy may be associated with lower mortality in patients with diastolic heart failure: a preliminary report. Circulation 2005; 112:357-63.

13 Ray JG, Gong Y, Sykora K, et al. Statin use and survival outcomes in elderly patients with heart failure. Arch Intern Med 2005; 165:62-7.

14 Sola S, Mir MQ, Lerakis S, et al. Atorvastatin improves left ventricular systolic function and serum markers of inflammation in nonischemic heart failure. J Am Coll Cardiol 2006;47:332-7.

15 Mozaffarian D, Minami E, Letterer RA, et al. The effects of atorvastatin (10 $\mathrm{mg}$ ) on systemic inflammation in heart failure. Am J Cardiol 2005:96:1699-704.

16 Bleske BE, Nicklas JM, Bard RL, et al. Neutral effect on markers of heart failure, inflammation, endothelial activation and function, and vagal tone after high-dose HMG-CoA reductase inhibition in non-diabetic patients with nonischemic cardiomyopathy and average low-density lipoprotein level. J Am Coll Cardiol 2006;47:338-41. 